PROCEEDINGS OF THE

AMERICAN MATHEMATICAL SOCIETY

Volume 126, Number 12, December 1998, Pages 3639-3645

S $0002-9939(98) 05028-\mathrm{X}$

\title{
WICK PRODUCTS OF THE CAR ALGEBRA
}

\author{
E. R. NEGRIN \\ (Communicated by Palle E. T. Jorgensen)
}

\begin{abstract}
The purpose of this paper is to put in a precise mathematical (algebraic) form the Wick products of the CAR algebra. We state in detail the reduction of the ordinary product of Fermi fields in terms of a finite sum of monomials in the creation and annihilation operators in which all creation operators occur to the left of all annihilation operators (Wick-ordered) and the Fock (vacuum) state of the former.
\end{abstract}

\section{INTRODUCTION}

Let $\mathbf{H}$ be a complex Hilbert space, and let $\mathcal{C}(\mathbf{H})$ be the CAR (canonical anticommutation relation) algebra over $\mathbf{H}$, i.e., the $C^{*}$-algebra generated by the set

$$
\left\{a^{*}(f), a(f) \mid f \in \mathbf{H}\right\},
$$

together with a unit $e$, and whose elements satisfy the anticommutation relations

$$
\begin{gathered}
a(f) a^{*}(g)+a^{*}(g) a(f)=\langle f, g\rangle e, \\
a^{*}(f) a^{*}(g)+a^{*}(g) a^{*}(f)=0, \\
a(f) a(g)+a(g) a(f)=0, \quad f, g \in \mathbf{H} .
\end{gathered}
$$

We will denote

$$
\psi(f)=a^{*}(f)+a(f), \quad f \in \mathbf{H} .
$$

As usual, we will refer to $\psi(f)$ as the Fermi field, and $a^{*}(f)$ and $a(f)$ as the creation and annihilation operators, respectively.

Here, we obtain an explicit expression for the reduction of the product $\psi\left(f_{1}\right) \ldots$ $\psi\left(f_{n}\right)$ in terms of Wick-ordered products, in which all creation operators occur to the left of all annihilation operators, that is, a finite linear combination of monomials of the form $a^{*}\left(f_{1}\right) \cdots a^{*}\left(f_{m}\right) a\left(g_{1}\right) \cdots a\left(g_{n}\right)$, for vectors $f_{i}, g_{j} \in \mathbf{H}$.

This program, developed first by F.J. Dyson [4] and G.C. Wick [9], has been useful in practical quantum mechanics, especially in applications to Feynman graph theory, where the Fermi field plays an important role in defining Feynman propagators as the Fock (vacuum) state of ordinary products of them (cf. [2, §17.4], [3, $\S 17]$ and $[5, \S 4-2]$.)

Our treatment benefits immeasurably from the interpretation on Wick products of Bose fields given by I.E. Segal (cf. [7], [8, §1.4]) and I.E. Segal et al. [1, §7.2].

Received by the editors November 19, 1996.

1991 Mathematics Subject Classification. Primary 81T08.

Key words and phrases. Wick-ordered, vacuum state, Fermi field, CAR algebra.

(C) 1998 American Mathematical Society 


\section{Algebraic Process of ReduCtion}

Definition 1. A Fock (vacuum) state on $\mathcal{C}(\mathbf{H})$ is a linear functional $E$ on $\mathcal{C}(\mathbf{H})$ such that $E(e)=1$ and

$$
\begin{gathered}
E\left(a^{*}\left(f_{1}\right) \cdots a^{*}\left(f_{m}\right) a\left(g_{1}\right) \cdots a\left(g_{n}\right)\right)=0, \\
E\left(a^{*}\left(f_{1}\right) \cdots a^{*}\left(f_{m}\right)\right)=0, \\
E\left(a\left(g_{1}\right) \cdots a\left(g_{n}\right)\right)=0,
\end{gathered}
$$

for all $f_{1}, \ldots, f_{m}$ and $g_{1}, \ldots, g_{n}$ in $\mathbf{H}$, and all $m, n \in \mathbb{N}$.

Note that, from [6, formula (2.5), p. 252] there exists a unique linear functional satisfying the preceding conditions.

An explicit mathematical definition of Wick-ordered products of the CAR algebra may be formulated as follows.

Definition 2. The Wick product of $\psi\left(f_{1}\right) \cdots \psi\left(f_{n}\right)$, with $f_{1}, \ldots, f_{n}$ in $\mathbf{H}$, is the element of $\mathcal{C}(\mathbf{H})$,

$$
: \psi\left(f_{1}\right) \cdots \psi\left(f_{n}\right):=\sum_{S \subseteq\{1, \ldots, n\}} \delta_{P}\left\{\prod_{k \in S} a^{*}\left(f_{k}\right)\right\}\left\{\prod_{k \in\{1, \ldots, n\}-S} a\left(f_{k}\right)\right\},
$$

where $\delta_{P}$ denotes the sign of the permutation needed to permute $1, \ldots, n$ into the order in which it appears in the formula, and the summation ranges over all subsets $S$ of $\{1, \ldots, n\}$.

Note that from relations (1.1), (1.2) and (1.3), the products involved in formula (2.1) are independent of the order of the factors. Thus, the Wick product $: \psi\left(f_{1}\right) \cdots \psi\left(f_{n}\right):$ is well defined.

The next result will be useful.

Lemma. For any $f_{1}, \ldots, f_{n}$ in $\mathbf{H}, n \in \mathbb{N}, n>1$, it follows that

$$
\begin{gathered}
: \psi\left(f_{1}\right) \cdots \psi\left(f_{n-1}\right): \psi\left(f_{n}\right)=: \psi\left(f_{1}\right) \cdots \psi\left(f_{n-1}\right) \psi\left(f_{n}\right): \\
+\sum_{1 \leq i \leq n-1} \delta_{i}: \psi\left(f_{1}\right) \cdots \widehat{\psi\left(f_{i}\right)} \cdots \psi\left(f_{n-1}\right): E\left(\psi\left(f_{i}\right) \psi\left(f_{n}\right)\right),
\end{gathered}
$$

where $\delta_{i}$ denotes the sign of the permutation $1, \ldots, \hat{i}, \ldots, n-1, i, n$.

Proof. We will prove this Lemma by induction on $n$. For $n=2$, it establishes that $\psi\left(f_{1}\right) \psi\left(f_{2}\right)=: \psi\left(f_{1}\right) \psi\left(f_{2}\right):+E\left(\psi\left(f_{1}\right) \psi\left(f_{2}\right)\right)$, which is easily seen to be valid by direct computations from (2.1). 
Now,

$$
\begin{aligned}
: \psi\left(f_{1}\right) \cdots \psi\left(f_{n-1}\right): \psi\left(f_{n}\right) & =\sum_{S \subseteq\{1, \ldots, n-1\}} \delta_{P}\left\{\prod_{k \in S} a^{*}\left(f_{k}\right)\right\}\left\{\prod_{k \in\{1, \ldots, n-1\}-S} a\left(f_{k}\right)\right\} \psi\left(f_{n}\right) \\
= & \sum_{S \subseteq\{1, \ldots, n-1\}} \delta_{P}\left\{\prod_{k \in S} a^{*}\left(f_{k}\right)\right\}\left\{\prod_{k \in\{1, \ldots, n-1\}-S} a\left(f_{k}\right)\right\}\left(a^{*}\left(f_{n}\right)+a\left(f_{n}\right)\right) \\
= & \sum_{S \subseteq\{1, \ldots, n-1\}} \delta_{P}\left\{\prod_{k \in S} a^{*}\left(f_{k}\right)\right\}\left\{\prod_{k \in\{1, \ldots, n-1\}-S} a\left(f_{k}\right)\right\} a^{*}\left(f_{n}\right) \\
& +\sum_{S \subseteq\{1, \ldots, n-1\}} \delta_{P}\left\{\prod_{k \in S} a^{*}\left(f_{k}\right)\right\}\left\{\begin{array}{l}
\left.\prod_{k \in\{1, \ldots, n-1\}-S} a\left(f_{k}\right)\right\} a\left(f_{n}\right) .
\end{array}\right.
\end{aligned}
$$

From relations (1.1), (1.2) and (1.3), the fact that $E\left(\psi\left(f_{1}\right) \psi\left(f_{2}\right)\right)=\left\langle f_{2}, f_{1}\right\rangle$ and the definition of $\delta_{P}$, formula (2.3) yields $(2.2)$.

With this result, the reduction is obtained in

Theorem. Let $\mathcal{C}(\mathbf{H})$ be the CAR algebra over a complex Hilbert space $\mathbf{H}$, and let $E$ be the Fock (vacuum) state on $\mathcal{C}(\mathbf{H})$. Then, for any $f_{1}, \ldots, f_{n}$ in $\mathbf{H}$,

$$
\begin{aligned}
& \psi\left(f_{1}\right) \cdots \psi\left(f_{n}\right)=: \psi\left(f_{1}\right) \cdots \psi\left(f_{n}\right): \\
& +\sum_{1 \leq j<k \leq n} \delta_{j k}: \psi\left(f_{1}\right) \cdots \widehat{\psi\left(f_{j}\right)} \cdots \widehat{\psi\left(f_{k}\right)} \cdots \psi\left(f_{n}\right): E\left(\psi\left(f_{j}\right) \psi\left(f_{k}\right)\right) \\
& +\sum_{1 \leq j_{1}<k_{1} \leq n, 1 \leq j_{2}<k_{2} \leq n} \delta_{j_{1} k_{1} j_{2} k_{2}}: \psi\left(f_{1}\right) \cdots \widehat{\psi\left(f_{j_{1}}\right)} \cdots \widehat{\psi\left(f_{k_{1}}\right)} \\
& \quad \cdots \widehat{\psi\left(f_{j_{2}}\right)} \cdots \widehat{\psi\left(f_{k_{2}}\right)} \cdots \psi\left(f_{n}\right): E\left(\psi\left(f_{j_{1}}\right) \psi\left(f_{k_{1}}\right)\right) E\left(\psi\left(f_{j_{2}}\right) \psi\left(f_{k_{2}}\right)\right) \\
& \quad+\cdots+\sum_{1 \leq j_{1}<k_{1} \leq n, \ldots, 1 \leq j_{s}<k_{s} \leq n} \delta_{j_{1} k_{1} \cdots j_{s} k_{s}} u E\left(\psi\left(f_{j_{1}}\right) \psi\left(f_{k_{1}}\right)\right) \cdots E\left(\psi\left(f_{j_{s}}\right) \psi\left(f_{k_{s}}\right)\right),
\end{aligned}
$$

where $s$ denotes the integral part of $n / 2$, and if $n$ is even $u=e$ (the identity in $\mathcal{C}(\mathbf{H})$ ), but if $n$ is odd $u=\psi\left(f_{t}\right)$, where $t$ is the unique integer such that $1 \leq t \leq n$ and $t \neq j_{m}, t \neq k_{m}$ for all $m=1, \ldots, s$.

In both cases, the sums are taken only over $j_{m}, k_{m}$ such that $j_{m} \neq j_{n}, j_{m} \neq k_{n}$ and $k_{m} \neq k_{n}$ for all $m, n \in \mathbb{N}$ with $m \neq n$. Moreover, the sums range only over $j_{1}<j_{2}<\cdots<j_{m}$.

Also, in both cases, $\delta_{i_{1} \ldots i_{m}}$ denotes the sign of the permutation

$$
1, \ldots, \widehat{i_{1}}, \ldots, \widehat{i_{m}}, \ldots, n, i_{1}, \ldots, i_{m} \text {. }
$$

Proof. We will prove this result by induction on $n$. Clearly $\psi\left(f_{1}\right)=: \psi\left(f_{1}\right):$. For the case when $n=2$, one has $\psi\left(f_{1}\right) \psi\left(f_{2}\right)=: \psi\left(f_{1}\right) \psi\left(f_{2}\right):+E\left(\psi\left(f_{1}\right) \psi\left(f_{2}\right)\right)$, which is easily seen to be valid. 
Assume $n$ is odd. By the induction hypothesis

$$
\begin{gathered}
\psi\left(f_{1}\right) \cdots \psi\left(f_{n-1}\right) \psi\left(f_{n}\right)=: \psi\left(f_{1}\right) \cdots \psi\left(f_{n-1}\right): \psi\left(f_{n}\right) \\
+\sum_{1 \leq j<k \leq n-1} \delta_{j k}^{\prime}: \psi\left(f_{1}\right) \cdots \widehat{\psi\left(f_{j}\right)} \cdots \widehat{\psi\left(f_{k}\right)} \cdots \psi\left(f_{n-1}\right): \psi\left(f_{n}\right) E\left(\psi\left(f_{j}\right) \psi\left(f_{k}\right)\right) \\
\quad+\sum_{1 \leq j_{1}<k_{1} \leq n-1,1 \leq j_{2}<k_{2} \leq n-1} \delta_{j_{1} k_{1} j_{2} k_{2}}^{\prime}: \psi\left(f_{1}\right) \cdots \widehat{\psi\left(f_{j_{1}}\right)} \cdots \widehat{\psi\left(f_{k_{1}}\right)} \\
\cdots \widehat{\psi\left(f_{j_{2}}\right)} \cdots \widehat{\psi\left(f_{k_{2}}\right)} \cdots \psi\left(f_{n-1}\right): \psi\left(f_{n}\right) E\left(\psi\left(f_{j_{1}}\right) \psi\left(f_{k_{1}}\right)\right) E\left(\psi\left(f_{j_{2}}\right) \psi\left(f_{k_{2}}\right)\right) \\
+\cdots+\sum_{1 \leq j_{1}<k_{1} \leq n-1, \ldots, 1 \leq j_{s_{*}}<k_{s_{*}} \leq n-1} \delta_{j_{1} k_{1} \ldots j_{s_{*}} k_{s_{*}}}^{\prime} \psi\left(f_{n}\right) E\left(\psi\left(f_{j_{1}}\right) \psi\left(f_{k_{1}}\right)\right) \\
\cdots E\left(\psi\left(f_{j_{s_{*}}}\right) \psi\left(f_{k_{s_{*}}}\right)\right),
\end{gathered}
$$

where $s_{*}$ denotes the integral part of $(n-1) / 2$, and $\delta_{i_{1} \ldots i_{m}}^{\prime}$ denotes the sign of the permutation

$$
1, \ldots, \widehat{i_{1}}, \ldots, \widehat{i_{m}}, \ldots, n-1, i_{1}, \ldots, i_{m} .
$$

Now, using the preceding Lemma, the equality above becomes

$$
\begin{aligned}
& \psi\left(f_{1}\right) \cdots \psi\left(f_{n-1}\right) \psi\left(f_{n}\right)=: \psi\left(f_{1}\right) \cdots \psi\left(f_{n-1}\right) \psi\left(f_{n}\right): \\
& +\sum_{1 \leq i \leq n-1} \delta_{i}^{i}: \psi\left(f_{1}\right) \cdots \widehat{\psi\left(f_{i}\right)} \cdots \psi\left(f_{n-1}\right): E\left(\psi\left(f_{i}\right) \psi\left(f_{n}\right)\right) \\
& +\sum_{1 \leq j<k \leq n-1} \delta_{j k}^{\prime}: \psi\left(f_{1}\right) \cdots \widehat{\psi\left(f_{j}\right)} \cdots \widehat{\psi\left(f_{k}\right)} \cdots \psi\left(f_{n}\right): E\left(\psi\left(f_{j}\right) \psi\left(f_{k}\right)\right) \\
& +\sum_{1 \leq j<k \leq n-1} \sum_{1 \leq i \leq n-1, i \neq j, i \neq k} \delta_{i}^{j k} \delta_{j k}^{\prime}: \psi\left(f_{1}\right) \cdots \widehat{\psi\left(f_{j}\right)} \cdots \widehat{\psi\left(f_{k}\right)} \cdots \widehat{\psi\left(f_{i}\right)} \cdots \psi\left(f_{n-1}\right): \\
& E\left(\psi\left(f_{j}\right) \psi\left(f_{k}\right)\right) E\left(\psi\left(f_{i}\right) \psi\left(f_{n}\right)\right) \\
& +\sum_{1 \leq j_{1}<k_{1} \leq n-1,1 \leq j_{2}<k_{2} \leq n-1} \delta_{j_{1} k_{1} j_{2} k_{2}}^{\prime}: \psi\left(f_{1}\right) \cdots \widehat{\psi\left(f_{j_{1}}\right)} \cdots \widehat{\psi\left(f_{k_{1}}\right)} \\
& \cdots \widehat{\psi\left(f_{j_{2}}\right)} \cdots \widehat{\psi\left(f_{k_{2}}\right)} \psi\left(f_{n-1}\right) \psi\left(f_{n}\right): E\left(\psi\left(f_{j_{1}}\right) \psi\left(f_{k_{1}}\right)\right) E\left(\psi\left(f_{j_{2}}\right) \psi\left(f_{k_{2}}\right)\right) \\
& +\sum_{\substack{1 \leq j_{1}<k_{1} \leq n-1 \\
1 \leq j_{2}<k_{2} \leq n-1}} \sum_{\substack{1 \leq i \leq n-1 \\
i \neq j_{1}, i \neq j_{2}, i \neq k_{2}}} \delta_{i}^{j_{1} k_{1} j_{2} k_{2}} \delta_{j_{1} k_{1} j_{2} k_{2}}^{\prime}: \psi\left(f_{1}\right) \cdots \widehat{\psi\left(f_{j_{1}}\right)} \\
& \cdots \widehat{\psi\left(f_{k_{1}}\right)} \cdots \widehat{\psi\left(f_{j_{2}}\right)} \cdots \widehat{\psi\left(f_{k_{2}}\right)} \cdots \widehat{\psi\left(f_{i}\right)} \cdots \psi\left(f_{n-1}\right) \text { : } \\
& E\left(\psi\left(f_{j_{1}}\right) \psi\left(f_{k_{1}}\right)\right) E\left(\psi\left(f_{j_{2}}\right) \psi\left(f_{k_{2}}\right)\right) E\left(\psi\left(f_{i}\right) \psi\left(f_{n}\right)\right) \\
& +\cdots+\sum_{\substack{1 \leq j_{1}<k_{1} \leq n-1, \ldots \\
1 \leq j_{s_{*}-1}<k_{s_{*}-1} \leq n-1}} \sum_{\substack{1 \leq i \leq n, i \neq j_{1} \\
i \neq k_{1}, \ldots, i \neq j_{s_{*}-1}, i \neq k_{s_{*}-1}}} \delta_{i}^{j_{1} k_{1} \ldots j_{s_{*}-1} k_{s_{*}-1}} \\
& \delta_{j_{1} k_{1} \ldots j_{s_{*}-1} k_{s_{*}-1}}^{\prime} \psi\left(f_{t}\right) E\left(\psi\left(f_{j_{1}}\right) \psi\left(f_{k_{1}}\right)\right) \cdots E\left(\psi\left(f_{j_{s_{*}-1}}\right) \psi\left(f_{k_{s_{*}-1}}\right)\right) E\left(\psi\left(f_{i}\right) \psi\left(f_{n}\right)\right) \\
& +\sum_{1 \leq j_{1}<k_{1} \leq n-1, \ldots, 1 \leq j_{s_{*}}<k_{s_{*}} \leq n-1} \delta_{j_{1} k_{1} \ldots j_{s_{*}} k_{s_{*}}}^{\prime} \psi\left(f_{n}\right) E\left(\psi\left(f_{j_{1}}\right) \psi\left(f_{k_{1}}\right)\right) \\
& \cdots E\left(\psi\left(f_{j_{s_{*}}}\right) \psi\left(f_{k_{s_{*}}}\right)\right),
\end{aligned}
$$


where $t$ is the unique integer such that $1 \leq t \leq n-1$ and $t \neq i, t \neq j_{m}, t \neq k_{m}$, $i \neq j_{m}, i \neq k_{m}$, for all $m=1, \ldots, s_{*}-1$ and $1 \leq i \leq n-1$. Moreover, $\delta_{i}^{i_{1} \ldots i_{m}}$ denotes the sign of the permutation

$$
1, \ldots, \widehat{i_{1}}, \ldots, \widehat{i_{m}}, \ldots, \widehat{i}, \ldots, n-1, i, n .
$$

With regard to the sums of each two consecutive terms in formula (2.4) one has

$$
\begin{gathered}
\sum_{1 \leq j_{1}<k_{1} \leq n-1, \ldots, 1 \leq j_{m}<k_{m} \leq n-1} \sum_{1 \leq i \leq n-1, i \neq j_{1}, i \neq k_{1}, \ldots, i \neq j_{m}, i \neq k_{m}} \delta_{i}^{j_{1} k_{1} \ldots j_{m} k_{m}} \delta_{j_{1} k_{1} \ldots j_{m} k_{m}}^{\prime} \\
: \psi\left(f_{1}\right) \cdots \widehat{\psi\left(f_{j_{1}}\right)} \cdots \widehat{\psi\left(f_{k_{1}}\right)} \cdots \widehat{\psi\left(f_{j_{m}}\right)} \cdots \widehat{\psi\left(f_{k_{m}}\right)} \cdots \widehat{\psi\left(f_{i}\right)} \cdots \psi\left(f_{n-1}\right): \\
E\left(\psi\left(f_{j_{1}}\right) \psi\left(f_{k_{1}}\right)\right) \cdots E\left(\psi\left(f_{j_{m}}\right) \psi\left(f_{k_{m}}\right)\right) E\left(\psi\left(f_{i}\right) \psi\left(f_{n}\right)\right) \\
\sum_{1 \leq j_{1}<k_{1} \leq n-1, \ldots, 1 \leq j_{m+1}<k_{m+1} \leq n-1} \delta_{j_{1} k_{1} \ldots j_{m+1} k_{m+1}}^{\prime}: \psi\left(f_{1}\right) \cdots \widehat{\psi\left(f_{j_{1}}\right)} \cdots \widehat{\psi\left(f_{k_{1}}\right)} \\
\cdots \psi \widehat{\left(f_{j_{m+1}}\right) \cdots \psi \widehat{\left(f_{k_{m+1}}\right)} \cdots \psi\left(f_{n}\right): E\left(\psi\left(f_{j_{1}}\right) \psi\left(f_{k_{1}}\right)\right) \cdots E\left(\psi\left(f_{j_{m+1}}\right) \psi\left(f_{k_{m+1}}\right)\right)} \\
=\sum_{1 \leq j_{1}<k_{1} \leq n, \ldots, 1 \leq j_{m}<k_{m} \leq n} \delta_{j_{1} k_{1} \ldots j_{m} k_{m}}: \psi\left(f_{1}\right) \cdots \widehat{\psi\left(f_{j_{1}}\right)} \cdots \widehat{\psi\left(f_{k_{1}}\right)} \cdots \widehat{\psi\left(f_{j_{m}}\right)} \\
\cdots \widehat{\psi\left(f_{k_{m}}\right)} \cdots \psi\left(f_{n}\right): E\left(\psi\left(f_{j_{1}}\right) \psi\left(f_{k_{1}}\right)\right) \cdots E\left(\psi\left(f_{j_{m}}\right) \psi\left(f_{k_{m}}\right)\right)
\end{gathered}
$$

Using this fact, one obtains the desired result for $n$ odd.

Now, assume $n$ is even. By the induction hypothesis,

$$
\begin{gathered}
\quad \psi\left(f_{1}\right) \cdots \psi\left(f_{n-1}\right) \psi\left(f_{n}\right)=: \psi\left(f_{1}\right) \cdots \psi\left(f_{n-1}\right): \psi\left(f_{n}\right) \\
+\sum_{1 \leq j<k \leq n-1} \delta_{j_{k}}^{\prime}: \psi\left(f_{1}\right) \cdots \widehat{\psi\left(f_{j}\right)} \cdots \widehat{\psi\left(f_{k}\right)} \cdots \psi\left(f_{n-1}\right): \psi\left(f_{n}\right) E\left(\psi\left(f_{j}\right) \psi\left(f_{k}\right)\right) \\
+\cdots+\sum_{1 \leq j_{1}<k_{1} \leq n-1,1 \leq j_{2}<k_{2} \leq n-1} \sum_{j_{1} k_{1} j_{2} k_{2}}^{\prime}: \psi\left(f_{1}\right) \cdots \widehat{\psi\left(f_{j_{1}}\right)} \cdots \widehat{\psi\left(f_{k_{1}}\right)} \cdots \widehat{\psi\left(f_{j_{2}}\right)} \cdots \widehat{\psi\left(f_{k_{2}}\right)} \\
+\cdots \psi\left(f_{n-1}\right): \psi\left(f_{n}\right) E\left(\psi\left(f_{j_{1}}\right) \psi\left(f_{k_{1}}\right)\right) E\left(\psi\left(f_{j_{2}}\right) \psi\left(f_{k_{2}}\right)\right) \\
\cdots E\left(\psi\left(f_{j_{1} k_{1} \cdots j_{s_{*}} k_{s_{*}}}\right) \psi\left(f_{t}\right) \psi\left(f_{n}\right) E\left(\psi\left(f_{k_{s_{*}}}\right)\right)\right.
\end{gathered}
$$

where $s_{*}$ denotes the integral part of $(n-1) / 2$ and $\delta_{i_{1} \ldots i_{m}}^{\prime}$ denotes the sign of the permutation

$$
1, \ldots, \widehat{i_{1}}, \ldots, \widehat{i_{m}}, \ldots, n-1, i_{1}, \ldots, i_{m} .
$$

Moreover $t$ is the unique integer such that $1 \leq t \leq n-1$, and $t \neq j_{m}, t \neq k_{m}$, for all $m=1, \ldots, s_{*}$.

Using again the preceding Lemma, the equality above becomes

$$
\begin{gathered}
\psi\left(f_{1}\right) \cdots \psi\left(f_{n-1}\right) \psi\left(f_{n}\right)=: \psi\left(f_{1}\right) \cdots \psi\left(f_{n-1}\right) \psi\left(f_{n}\right): \\
+\sum_{1 \leq i \leq n-1} \delta_{i}^{i}: \psi\left(f_{1}\right) \cdots \widehat{\psi\left(f_{i}\right)} \cdots \psi\left(f_{n-1}\right): E\left(\psi\left(f_{i}\right) \psi\left(f_{n}\right)\right) \\
+\sum_{1 \leq j<k \leq n-1} \delta_{j k}^{\prime}: \psi\left(f_{1}\right) \cdots \widehat{\psi\left(f_{j}\right)} \cdots \widehat{\psi\left(f_{k}\right)} \cdots \psi\left(f_{n-1}\right) \psi\left(f_{n}\right): E\left(\psi\left(f_{j}\right) \psi\left(f_{k}\right)\right) \\
+\sum_{1 \leq j<k \leq n-1} \sum_{1 \leq i \leq n-1, i \neq j, i \neq k} \delta_{i}^{j k} \delta_{j k}^{\prime}: \psi\left(f_{1}\right) \cdots \widehat{\psi\left(f_{j}\right)} \cdots \widehat{\psi\left(f_{k}\right)} \cdots \widehat{\psi\left(f_{i}\right)} \cdots \psi\left(f_{n-1}\right): \\
E\left(\psi\left(f_{j}\right) \psi\left(f_{k}\right)\right) E\left(\psi\left(f_{i}\right) \psi\left(f_{n}\right)\right)
\end{gathered}
$$




$$
\begin{aligned}
& 3644 \quad \text { E. R. NEGRIN } \\
& +\sum_{1 \leq j_{1}<k_{1} \leq n-1,1 \leq j_{2}<k_{2} \leq n-1} \delta_{j_{1} k_{1} j_{2} k_{2}}^{\prime}: \psi\left(f_{1}\right) \cdots \widehat{\psi\left(f_{j_{1}}\right)} \cdots \widehat{\psi\left(f_{k_{1}}\right)} \cdots \widehat{\psi\left(f_{j_{2}}\right)} \cdots \widehat{\psi\left(f_{k_{2}}\right)} \\
& \cdots \psi\left(f_{n-1}\right) \psi\left(f_{n}\right): E\left(\psi\left(f_{j_{1}}\right) \psi\left(f_{k_{1}}\right)\right) E\left(\psi\left(f_{j_{2}}\right) \psi\left(f_{k_{2}}\right)\right) \\
& +\sum_{1 \leq j_{1}<k_{1} \leq n-1,1 \leq j_{2}<k_{2} \leq n-1} \sum_{1 \leq i \leq n-1, i \neq j_{1}, i \neq k_{1}, i \neq j_{2}, i \neq k_{2}} \delta_{i}^{j_{1} k_{1} j_{2} k_{2}} \delta_{j_{1} k_{1} j_{2} k_{2}}^{\prime} \\
& : \psi\left(f_{1}\right) \cdots \widehat{\psi\left(f_{j_{1}}\right)} \cdots \widehat{\psi\left(f_{k_{1}}\right)} \cdots \widehat{\psi\left(f_{j_{2}}\right)} \cdots \widehat{\psi\left(f_{k_{2}}\right)} \cdots \widehat{\psi\left(f_{i}\right)} \cdots \psi\left(f_{n-1}\right): \\
& E\left(\psi\left(f_{j_{1}}\right) \psi\left(f_{k_{1}}\right)\right) E\left(\psi\left(f_{j_{2}}\right) \psi\left(f_{k_{2}}\right)\right) E\left(\psi\left(f_{i}\right) \psi\left(f_{n}\right)\right) \\
& +\cdots+\sum_{1 \leq j_{1}<k_{1} \leq n-1, \ldots, 1 \leq j_{s_{*}-1}<k_{s_{*}-1} \leq n-1} \sum_{1 \leq i \leq n-1, i \neq j_{1}, i \neq k_{1}, \ldots, i \neq j_{s_{*}-1}, i \neq k_{s_{*}-1}} \\
& \delta_{i}^{j_{1} k_{1} \ldots j_{s_{*}-1} k_{s_{*}-1}} \delta_{j_{1} k_{1} \ldots j_{s_{*}-1} k_{s_{*}-1}}^{\prime}: \psi\left(f_{p}\right) \psi\left(f_{q}\right): E\left(\psi\left(f_{j_{1}}\right) \psi\left(f_{k_{1}}\right)\right) \\
& \cdots E\left(\psi\left(f_{j_{s_{*}-1}}\right) \psi\left(f_{k_{s_{*}-1}}\right)\right) E\left(\psi\left(f_{i}\right) \psi\left(f_{n}\right)\right) \\
& +\sum_{1 \leq j_{1}<k_{1} \leq n-1, \ldots, 1 \leq j_{s_{*}}<k_{s_{*}} \leq n-1} \delta_{j_{1} k_{1} \ldots j_{s_{*}} k_{s_{*}}}^{\prime} \\
& \psi\left(f_{t}\right) \psi\left(f_{n}\right) E\left(\psi\left(f_{j_{1}}\right) \psi\left(f_{k_{1}}\right)\right) \cdots E\left(\psi\left(f_{j_{s_{*}}}\right) \psi\left(f_{k_{s_{*}}}\right)\right),
\end{aligned}
$$

where $p$ and $q$ denote the unique integers $1 \leq p<q \leq n-1$, such that $p \neq i$, $q \neq i, p \neq j_{m}, q \neq k_{m}, i \neq j_{m}, i \neq k_{m}$, for all $m=1, \ldots s_{*}-1$ and $1 \leq i \leq n-1$. Moreover, $\delta_{i}^{i_{1} \ldots i_{m}}$ denotes the sign of the permutation

$$
1, \ldots, \widehat{i_{1}}, \ldots, \widehat{i_{m}}, \ldots, \widehat{i}, \ldots, n-1, i, n .
$$

Now, arguing as in the case when $n$ is odd, using the fact that $\psi\left(f_{t}\right) \psi\left(f_{n}\right)=$ $: \psi\left(f_{t}\right) \psi\left(f_{n}\right):+E\left(\psi\left(f_{t}\right) \psi\left(f_{n}\right)\right)$ into formula (2.5), and taking into account the sign of the permutation, one obtains the desired result for $n$ even.

As a straightforward consequence of this result we have the following

Corollary. Let $\mathcal{C}(\mathbf{H})$ be the $C A R$ algebra over a complex Hilbert space $\mathbf{H}$, and let $E$ be the Fock (vacuum) state on $\mathcal{C}(\mathbf{H})$. Then, for any $f_{1}, \ldots, f_{n}$ in $\mathbf{H}$, one has:

a) If $n$ is odd, $E\left(\psi\left(f_{1}\right) \cdots \psi\left(f_{n}\right)\right)=0$.

b) If $n$ is even,

$$
=\sum_{1 \leq j_{1}<k_{1} \leq n, \ldots, 1 \leq j_{s}<k_{s} \leq n} E\left(\psi\left(f_{1}\right) \cdots \psi\left(f_{n}\right)\right)
$$

where the sums, $s$ and $\delta$ are defined as in the Theorem above.

Remark. It is clarifying to compute directly from (2.1) the following expressions (which agree with the formulae obtained in the Theorem and Corollary above):

and

$$
\begin{gathered}
\psi\left(f_{1}\right) \psi\left(f_{2}\right) \psi\left(f_{3}\right)=: \psi\left(f_{1}\right) \psi\left(f_{2}\right) \psi\left(f_{3}\right):+\psi\left(f_{1}\right) E\left(\psi\left(f_{2}\right) \psi\left(f_{3}\right)\right) \\
-\psi\left(f_{2}\right) E\left(\psi\left(f_{1}\right) \psi\left(f_{3}\right)\right)+\psi\left(f_{3}\right) E\left(\psi\left(f_{1}\right) \psi\left(f_{2}\right)\right),
\end{gathered}
$$

$$
\begin{gathered}
\psi\left(f_{1}\right) \psi\left(f_{2}\right) \psi\left(f_{3}\right) \psi\left(f_{4}\right)=: \psi\left(f_{1}\right) \psi\left(f_{2}\right) \psi\left(f_{3}\right) \psi\left(f_{4}\right):+: \psi\left(f_{1}\right) \psi\left(f_{2}\right): E\left(\psi\left(f_{3}\right) \psi\left(f_{4}\right)\right) \\
-: \psi\left(f_{1}\right) \psi\left(f_{3}\right):=E\left(\psi\left(f_{2}\right) \psi\left(f_{4}\right)\right)+: \psi\left(f_{1}\right) \psi\left(f_{4}\right): E\left(\psi\left(f_{2}\right) \psi\left(f_{3}\right)\right) \\
\quad+: \psi\left(f_{2}\right) \psi\left(f_{3}\right): E\left(\psi\left(f_{1}\right) \psi\left(f_{4}\right)\right)-: \psi\left(f_{2}\right) \psi\left(f_{4}\right): E\left(\psi\left(f_{1}\right) \psi\left(f_{3}\right)\right) \\
\quad+: \psi\left(f_{3}\right) \psi\left(f_{4}\right): E\left(\psi\left(f_{1}\right) \psi\left(f_{2}\right)\right)+E\left(\psi\left(f_{1}\right) \psi\left(f_{2}\right)\right) E\left(\psi\left(f_{3}\right) \psi\left(f_{4}\right)\right) \\
-E\left(\psi\left(f_{1}\right) \psi\left(f_{3}\right)\right) E\left(\psi\left(f_{2}\right) \psi\left(f_{4}\right)\right)+E\left(\psi\left(f_{1}\right) \psi\left(f_{4}\right)\right) E\left(\psi\left(f_{2}\right) \psi\left(f_{3}\right)\right) .
\end{gathered}
$$


For the Fock (vacuum) state values we obtain

$$
E\left(\psi\left(f_{1}\right) \psi\left(f_{2}\right) \psi\left(f_{3}\right)\right)=0,
$$

and

$$
\begin{gathered}
E\left(\psi\left(f_{1}\right) \psi\left(f_{2}\right) \psi\left(f_{3}\right) \psi\left(f_{4}\right)\right)=E\left(\psi\left(f_{1}\right) \psi\left(f_{2}\right)\right) E\left(\psi\left(f_{3}\right) \psi\left(f_{4}\right)\right) \\
-E\left(\psi\left(f_{1}\right) \psi\left(f_{3}\right)\right) E\left(\psi\left(f_{2}\right) \psi\left(f_{4}\right)\right)+E\left(\psi\left(f_{1}\right) \psi\left(f_{4}\right)\right) E\left(\psi\left(f_{2}\right) \psi\left(f_{3}\right)\right) .
\end{gathered}
$$

\section{REFERENCES}

[1] J. C. Baez, I. E. Segal and Z. Zhou, "Introduction to Algebraic and Constructive Quantum Field Theory", Princeton Univ. Press, Princeton, NJ, 1992.

[2] J. D. Bjorken and S. D. Drell, "Relativistic Quantum Fields", McGraw-Hill, NY, 1965. MR 32:5092

[3] N. N. Bogoliubov and D. V. Shirkov, "Quantum Fields", Benjamin/Cummings Pub. Co., Reading, MA, 1983. MR 85g:81096

[4] F. J. Dyson, The Radiation Theories of Tomonaga, Schwinger, and Feynman, Phys. Rev. (3) 75 (1949), 486-502. MR 10:418a

[5] C. Itzykson and J.-B. Zuber, "Quantum Field Theory", McGraw-Hill, NY, 1980. MR 82h:81002

[6] P. E. T. Jorgensen and G. L. Price, Index Theory and Second Quantization of Boundary Value Problems, J. Funct. Anal. 104 (1992), 243-290.

[7] I. E. Segal, Quantized Differential Forms, Topology 7 (1968), 147-172. MR 38:1113

[8] I. E. Segal, Nonlinear Functions of Weak Processes I, J. Funct. Anal. 4 (1969), 404-456. MR 40:2309

[9] G. C. Wick, The evaluation of the collision matrix, Phys. Rev. (2) 80 (1950), 268-272. MR 12:380d

Departamento de Análisis Matemático, Universidad de La Laguna, 38271 Canary IsLANDS, SPAIN

E-mail address: enegrin@ull.es 